\title{
The Effects of Polycations on Vascular Permeability in the Rat \\ A Proposed Role for Charge Sites
}

\author{
V. Matti Vehaskari, Charles T.-C. Chang, John K. Stevens, \\ and Alan M. Robson \\ Division of Nephrology, Edward Mallinckrodt Department of \\ Pediatrics, Washington University, the Renal Division, St. Louis \\ Children's Hospital, St. Louis, Missouri 63110
}

bstract. This study investigated whether charge sites in the walls of the microvasculature may play a role in maintaining the impermeability of the nonrenal capillaries to albumin. All experiments were performed in nephrectomized rats, studied in the awake state. The intravenous injection of protamine sulfate $(4 \mathrm{mg} / 100 \mathrm{~g}$ body wt dissolved in $0.9 \%$ saline) was followed by a mean increase of $29.1 \%$ in hematocrit and a decrease of $28.4 \%$ in plasma albumin concentration over a 10 -min period, indicating a significant $50-60 \%$ loss of albumin from the vascular space; a finding confirmed by studies using exogenous ${ }^{125}$ I-labeled albumin. Changes persisted for the remaining $80 \mathrm{~min}$ of observation, and could be reproduced by the injection of two other polycations, hexadimethrine and poly-l-lysine. These effects were not prevented by the antihistamine diphenhydramine hydrochloride. In contrast to ${ }^{125} \mathrm{I}$-labeled albumin, ${ }^{14} \mathrm{C}$-labeled neutral dextran of comparable size was not confined to the vascular space; its apparent volume of distribution progressively increased during the $90 \mathrm{~min}$ of observation. Intravenous injection of protamine sulfate was followed by a significantly smaller loss of ${ }^{14} \mathrm{C}$-dextran $(36.5 \%)$ than albumin $(59.1 \%)$ from the vascular space $(P<0.01)$. Protamine sulfate could not be demonstrated to result in any changes in the physicochemical characteristics of albumin. These observations suggest that the negative charge sites present in nonglomerular capillary walls have functions similar to equivalent sites present in the glomerular capillaries. Thus, charge sites could contribute to the low permeability of the microvasculature to neg-

Received for publication 4 January 1983 and in revised form 29 December 1983.

J. Clin. Invest.

(c) The American Society for Clinical Investigation, Inc.

$0021-9738 / 84 / 04 / 1053 / 09 \quad \$ 1.00$

Volume 73, April 1984, 1053-1061 atively charged macromolecules such as albumin. This may be an important mechanism for retaining albumin in the vascular space and preventing edema formation in health.

\section{Introduction}

Movement of fluid and solutes between plasma and interstitial fluid occurs both by bulk flow and by diffusion across the capillary walls (1). The major forces responsible for such movement are still thought to be those described by Starling in 1896, namely the sum of net hydrostatic and oncotic pressures (2). The absolute amount of water or solute that crosses the capillary wall is affected also by surface area of the capillary and by several membrane characteristics, which include hydraulic conductivity, and by both the diffusion and solvent drag reflection coefficients for the individual solute $(1,3-5)$.

The size of a molecule is important in determining the ability of that molecule to cross the capillary wall. For example, the permeability of muscle capillaries to albumin approximates only $0.33 \%$ that of the much smaller inulin molecule and $<0.01 \%$ that of water (1). Recent studies, however, have demonstrated that the conventional mathematical formulas that have beer developed to describe permeability of the microvasculature do not always explain experimental data (6). Such observations have raised the possibility that factors in addition to the traditional ones may need to be included in equations that relate the transvascular movement of solutes, especially proteins, to the primary driving forces.

Forces similar to those described for peripheral capillaries are responsible for the generation of glomerular filtrate (7). The functional characteristics of the glomerular capillaries, however, differ from those in other capillary beds. In addition, pore density and the hydrostatic pressure to which the glomerular capillaries are exposed are much higher. For these, and perhaps for other reasons, hydraulic permeability and the rate of fluid movement across the glomerular capillary is an order of magnitude greater than that observed in other capillaries (8). Despite these differences, the glomerular capillaries are remarkably impermeable to proteins (9). It has been proposed that this is due to the 
presence in the glomerular capillary walls of negatively charged molecules such as glycosaminoglycans (10), which provide an electrical barrier to supplement the mechanical ones and prevent the passage into the glomerular filtrate of proteins such as albumin, which are negatively charged at $\mathrm{pH} 7.4(11,12)$. Many observations support this concept. As reviewed elsewhere (12), negatively charged molecules are less able to penetrate the glomerular barrier and have a lower clearance than do uncharged or positively charged molecules of comparable size. Charge discrimination is lost in disease states associated with loss of glomerular polyanion (13). In minimal change nephrotic syndrome in man, a lesion characterized by highly selective proteinuria the albuminuria is attributable to an $\sim 50 \%$ reduction in the concentration of fixed negative charges in the glomerular capillary wall (14). Finally, intravenous or intrarenal arterial injection of polycations is followed by neutralization of glomerular charge and by a marked increase in albuminuria (15).

The present study was undertaken to determine whether charge may play an equally important role in determining permeability to protein in the nonrenal capillary beds. The results suggest that this is the case. Thus, electrical charge of both the microvascular wall and the circulating proteins may need to be incorporated into the mathematical formulas that describe fluid and solute fluxes across capillary walls (6).

\section{Methods}

All experiments were performed on young female Sprague-Dawley rats ranging in body weight from 210 to $350 \mathrm{~g}$. On the day of the experiment, the animal was weighed and a bilateral nephrectomy was performed through a midline abdominal incision using light ether anesthesia. The wound was then closed and one femoral artery was cannulated for sampling of arterial blood. To replace any surgical blood or fluid loss, a bolus of $1 \mathrm{ml}$ of $0.9 \%$ saline was given through a tail vein cannula, which was left in place for injection of test substances. Both cannulae were filled with heparin-saline ( $10 \mathrm{U}$. S. Pharmacopoeia $\mathrm{U} / \mathrm{ml}$ ) to maintain patency until subsequent use. After surgery, the animals were transferred into a bivalved plastic cylinder where they were restrained in an upright position and allowed to recover from the anesthetic. The methodologies used for these procedures were identical to those reported previously (16) except that in the present studies the animals' body temperatures were maintained by placing a heating pad under the operating table and around the plastic cylinder used for the physiologic studies.

Protocol $A$. The rats were allowed to recover from the anesthetic for $90 \mathrm{~min}$. At this time, $0.15-0.2 \mathrm{ml}$ blood was collected into heparinized capillary tubes from the femoral artery catheter. The arterial cannula was refilled with the heparinized saline and a volume of $0.9 \%$ saline, equal to the volume of blood withdrawn, was injected through the tail vein catheter. Further arterial blood samples were obtained in similar fashion 10, 20, and $28 \mathrm{~min}$ later. At $30 \mathrm{~min}$, each animal was given an intravenous injection of either $0.9 \%$ saline or a polycation dissolved in $0.9 \%$ saline. The volume of injectate in every animal was $0.4 \mathrm{ml} / 100$ $\mathrm{g}$ body wt. The polycations used were either protamine sulfate (Eli Lilly, Indianapolis, IN) given in a dose of $4 \mathrm{mg} / 100 \mathrm{~g}$ body wt; poly-l-lysine, $3,400 \mathrm{~mol} \mathrm{wt}$; (Sigma Chemical Co., St. Louis, MO) given in a dose of $1.5 \mathrm{mg} / 100 \mathrm{~g}$ body $\mathrm{wt}$; or hexadimethrine (Sigma Chemical Co.) given in a dose of $4 \mathrm{mg} / 100 \mathrm{~g}$ body wt. Further arterial blood samples were obtained at 32, 35, 38, 41, 50, and $60 \mathrm{~min}$. In representative animals, additional samples were obtained at 70,80 , and $90 \mathrm{~min}$. Animals were then killed and reweighed to ensure fluid balance had been maintained throughout the study.

Each blood sample was centrifuged and its hematocrit determined. In all of the animals injected with hexadimethrine, or poly-l-lysine, in 10 of the 17 injected with protamine sulfate, and in 12 of the 21 injected with saline, plasma albumin concentration of each sample was measured, in duplicate, by radial immunodiffusion $(17,18)$.

An additional group of rats was prepared as described. $60 \mathrm{~min}$ after surgery was completed an intravenous infusion of diphenhydramine hydrochloride, $(10 \mathrm{mg} / \mathrm{ml}$; Benadryl, Parke-Davis, Morris Plains, NJ) was begun and continued for $75 \mathrm{~min}$ at a rate calculated to provide a total dose of $25 \mathrm{mg} / \mathrm{kg}$ body wt. The remaining details of the study were identical to those already described so that the Benadryl infusion was begun $30 \mathrm{~min}$ before the first blood sample was obtained and was continued throughout the control period and the first $15 \mathrm{~min}$ of the experimental period. Protamine sulfate was the test substance injected in seven of these animals and normal saline was injected in the remaining five animals. Hematocrits and plasma albumin concentrations were measured in all of the blood samples obtained from these 12 animals.

Protocol $B$. Rats were prepared as described already. They were given an intravenous injection of a known amount of ${ }^{125}$ I-labeled albumin after they had been placed in the restraining cylinder. An arterial blood sample was then obtained $60 \mathrm{~min}$ later, after which the rat was given an intravenous injection of either saline or protamine sulfate dissolved in saline, as described previously. Further blood samples were obtained $2,5,8,11,20$, and 30 min after this injection. Hematocrit was measured in each blood sample and duplicate, measured volumes of plasma were counted for ${ }^{125} \mathrm{I}$-albumin in a gamma-spectrometer (model 5219, Packard Instruments Co., Inc., Downer's Grove, IL).

For these studies, rat albumin (Cappel Laboratories, Cochranville, PA) was labeled with ${ }^{125} \mathrm{I}$ with the iodogen method (19). 0.1-0.2 $\mu \mathrm{g}$ of the labeled albumin and $1 \mu \mathrm{g}$ of unlabeled albumin were diluted to 1 $\mathrm{ml}$ with $0.9 \%$ saline in a $1-\mathrm{ml}$ syringe. Each syringe was counted before and after its contents were injected into the rat to determine the amount of labeled albumin given. The dose injected averaged 300,000 (range 200,000-600,000) cpm.

Protocol C. Animals were prepared as in protocol A. After they had been in the restrainer for $90 \mathrm{~min}$ after surgery, they were given an intravenous injection of a known amount of ${ }^{14} \mathrm{C}$-labeled neutral dextran that had a molecular radius of $36 \AA$. Blood samples were obtained 30 , 40,50 , and $58 \mathrm{~min}$ later. At $60 \mathrm{~min}$, the rats were given an intravenous injection of either saline or protamine sulfate in saline as described already and further blood samples were obtained $2,5,8,11,20$, and $30 \mathrm{~min}$ later. After hematocrit determination, measured volumes of plasma (30-60 $\mu \mathrm{l})$ were added in duplicate to $10 \mathrm{ml}$ of a standard liquid scintillation fluid (16) containing $100 \mathrm{ml} /$ liter Bio-solv 3 (Beckman Instruments, Inc., Fullerton, CA) and were counted for ${ }^{14} \mathrm{C}$-activity in a liquid scintillation counter (model 3375, Packard Instrument Co., Inc.). Preliminary studies demonstrated that the volume of plasma used did not result in any quenching in the scintillator system.

The dextran for these studies was prepared from $\left[\right.$ carboxyl $\left[{ }^{14} \mathrm{C}\right]-$ dextran with an average molecular weight of 70,000 D and specific activity averaging $0.1 \mathrm{mCi} / 100 \mathrm{mg}$ (New England Nuclear, Boston, MA). This preparation was subjected to column chromatography with Sephacryl S-200 (Pharmacia Fine Chemicals, Piscataway, NJ). 0.9\% saline was used as the eluant. The dextran fraction that had an identical elution volume to albumin on this initial separation was collected and subjected 
to the column again with identical methods. The dextran fraction that was eluted from this second separation in the same volume as albumin was used in the animal studies. A known amount of this preparation (average $250,000 \mathrm{cpm}$ in a volume of $0.5 \mathrm{ml}$ ) was injected intravenously into each of the rats. The exact amount injected was determined by weighing the syringe before and after injection and by counting an aliquot of the injectate.

Calculations. The amount of albumin, ${ }^{125} \mathrm{I}$-albumin, or ${ }^{14} \mathrm{C}$-dextran remaining in the plasma at time $t$ after the injection of the polycation or saline $\left(A_{\mathfrak{t}}\right)$ was compared with the amount present just before the injection $\left(A_{0}\right)$ using the following formula:

$$
\frac{A_{\mathrm{t}}}{A_{0}}=\frac{C_{\mathrm{t}}\left(1-H c t_{\mathrm{t}}\right) H c t_{0}}{C_{0}\left(1-H c t_{0}\right) H c t_{\mathrm{t}}} \cdot \mathrm{q}_{\mathrm{t}},
$$

where $C_{0}, C_{\mathrm{t}}, H c t_{0}$, and $H c t_{\mathrm{t}}$ are the concentrations of either albumin or dextran, and the hematocrits just before and at time $t$ after the injection. As described below, $q_{t}$ corrects for reduction in erythrocyte mass due to repeated sampling of blood throughout the study.

This formula was derived as follows: the amount of albumin or dextran present in the plasma $(A)$ equals its concentration $(C)$ multiplied by plasma volume $(P V)$, or

$$
A=C(\mathrm{P} V) .
$$

Since $\mathrm{P} V=\mathrm{B} V(1-H c t)$, where $\mathrm{BV}$ equals blood volume and Hct, hematocrit; and $\mathrm{B} V=\mathrm{RBC} / H c t$, where $\mathrm{RBC}$ equals erythrocyte mass. Then Eq. 2 can be rewritten: $A=C(1-H c t)(\mathrm{RBC}) /(H c t)$.

The amounts of albumin or dextran remaining in the plasma space at time $t$ after the injection of the polycation or saline $\left(A_{t}\right)$ can thus be expressed as a fraction of the amount present before the injection $\left(A_{0}\right)$ as follows:

$$
\frac{A_{\mathrm{t}}}{A_{0}}=\frac{C_{\mathrm{t}}\left(1-H c t_{\mathrm{t}}\right)\left(\mathrm{RBC}_{\mathrm{t}}\right) /\left(H c t_{\mathrm{t}}\right)}{C_{0}\left(1-H c t_{0}\right)\left(\mathrm{RBC}_{0}\right) /\left(H c t_{0}\right)} .
$$

Because of repeated blood sampling, the erythrocyte mass decreased throughout the experiment in each animal. An estimate for the proportion of erythrocyte mass remaining in circulation at time $t\left(\mathrm{q}_{\mathrm{t}}\right)$ was derived from the ratio of the cumulative volume of blood sampled by time $t$ $\left(\mathrm{S}_{1}\right)$ and the animal's original blood volume $\left(\mathrm{B} V_{0}\right)$, which equals 0.058 $\times$ body wt in kilograms $(20)$ : $q_{t}=1-\left(S_{t}\right) /\left(B V_{0}\right)$. Thus, $R B C_{t}=q_{t}$ $\times R_{B C}$. Substituting $\mathrm{q}_{\mathrm{t}} \times \mathrm{RBC}_{0}$ for $R B C_{1}$ into Eq. 3, the values for $\mathrm{RBC}_{0}$ cancel out and this equation can be rewritten as Eq. 1 .

If erythrocyte volume changed during the study, it too would affect Eq. 1 by altering the relationship between erythrocyte mass and hematocrit. This was evaluated in a series of rats that were studied as outlined in protocol A. A single blood sample was obtained in the control period and two samples were collected during the experimental period, 15 and $30 \mathrm{~min}$ after injections of the test substance, either normal saline or protamine sulfate. Erythrocyte volume was measured in those samples with a Coulter counter (Coulter Electronics, Hialeah, FL). Mean erythrocyte volume during control periods was $59.9 \pm 2.0 \mu \mathrm{M}^{3}(n=6)$. Values did not change during the experimental periods. They were $60.3 \pm 2.0 \mu \mathrm{M}^{3}$ in the three rats injected with normal saline and $60.3 \pm 1.4$ $\mu \mathrm{M}^{3}$ in the three rats given protamine sulfate. Values at 15 and $30 \mathrm{~min}$ were not different. Thus, it was not necessary to incorporate values for erythrocyte volume into Eq. 1 .

Characterization of proteins. Animals were prepared and studied as described in protocol A, except only two blood samples were obtained in the control period. The animals were then injected with protamine sulfate as described and two further blood samples were obtained 30 and $60 \mathrm{~min}$ later. The samples were subjected to slab gel electrophoresis both in $10 \%$ polyacrylamide gel (21) and in the same gel containing $0.3 \%$ sodium dodecyl sulfate (SDS) (22). The slabs were then stained for identification and location of the protein bands $(21,22)$. Purified rat serum albumin (Cappel Laboratories), or the same albumin to which protamine sulfate had been added in a ratio of $5 \mathrm{mg}$ protamine sulfate/ $1 \mathrm{mg}$ albumin, was also subjected to both types of electrophoresis. Finally, rat serum albumin $(50 \mathrm{mg})$, either alone or mixed with protamine sulfate as already described, was subjected to column chromatography with Sephacryl S-200 in $2.6 \times 160-\mathrm{cm}$ columns. Effluent was monitored for absorption at $280 \mathrm{~nm}$ to identify the volume of distribution in which the albumin was eluted.

Statistical analyses. All values in the text and tables are expressed as mean $\pm \mathrm{SD}$; those in the figures as mean $\pm \mathrm{SEM}$. Data were evaluated for statistical significance by analysis of variance with repeated measures (23). Unless stated otherwise, all values for $P$ were derived from these calculations. Findings were confirmed by Hotelling's $T^{2}(23)$. Other statistical techniques such as regression analysis or the $t$ test were performed by standard methods (24).

\section{Results}

Hematocrit. During the 30-min control period in which blood was sampled on four occasions and replaced by saline, the animals' hematocrits fell slowly but progressively (Fig. 1). In the 21 control rats, the intravenous injection of vehicle (saline) was associated with a further abrupt but small decline in hematocrit followed by a continuing fall during the subsequent $30-\mathrm{min}$ of sampling. The hematocrit fell from $45.0 \pm 3.1 \%$ at the beginning of the control period to $40.6 \pm 3.5 \% 1 \mathrm{~h}$ later at the end of the study. In contrast, the injection of protamine sulfate was followed by a rapid and marked increase in hematocrit. In the 17 animals given this polycation, hematocrit averaged $43.7 \pm 2.6 \%$ immediately before the injection. It increased to $45.1 \pm 3.7 \% 2 \mathrm{~min}$ after the injection and rose progressively to a peak of $57.4 \pm 5.6 \%$

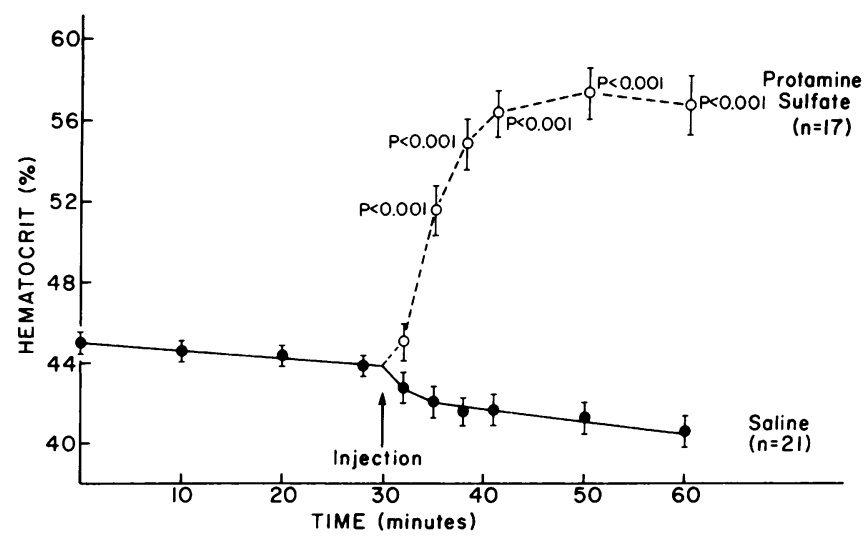

Figure 1. A comparison of the effects of injecting either saline or protamine sulfate on hematocrit. All values are shown as mean $\pm \mathrm{SEM}$. Values for $P$ were calculated by comparing the two results at each time interval using the $t$ test. $n$ indicates the number of animals studied. 
Table I. Effect on Hematocrit of an Intravenous Injection of either Saline or a Polycation in Nephrectomized Rats

\begin{tabular}{|c|c|c|c|c|c|c|c|c|}
\hline & \multirow[b]{2}{*}{ Preinjection* } & \multicolumn{6}{|c|}{ Hematocrit (minutes after injection) } & \multirow[b]{2}{*}{$P \ddagger$} \\
\hline & & 2 & 5 & 8 & 11 & 20 & 30 & \\
\hline & & $\%$ & \% & $\%$ & $\%$ & $\%$ & $\%$ & \\
\hline Saline $(n=21)$ & $44.3 \pm 3.1$ & $42.8 \pm 3.7$ & $42.1 \pm 3.7$ & $41.6 \pm 3.1$ & $41.7 \pm 3.5$ & $41.3 \pm 3.5$ & $40.6 \pm 3.5$ & \\
\hline Protamine sulfate $(n=17)$ & $44.0 \pm 2.6$ & $45.1 \pm 3.7$ & $51.6 \pm 5.3$ & $54.9 \pm 5.4$ & $56.4 \pm 5.0$ & $57.4 \pm 5.6$ & $56.8 \pm 6.2$ & $<0.0001$ \\
\hline Hexadimethrine $(n=6)$ & $45.8 \pm 3.3$ & $42.9 \pm 2.6$ & $50.3 \pm 4.7$ & $53.8 \pm 5.3$ & $53.8 \pm 5.4$ & $56.3 \pm 3.6$ & $57.3 \pm 1.7$ & $<0.0001$ \\
\hline Poly-l-lysine $(n=5)$ & $46.8 \pm 3.0$ & $50.9 \pm 1.5$ & $59.0 \pm 7.3$ & $58.9 \pm 4.6$ & $57.2 \pm 2.7$ & $57.5 \pm 3.1$ & $57.8 \pm 2.9$ & $<0.0001$ \\
\hline
\end{tabular}

Values are given in mean \pm SD. * Values were obtained 2 min before injection of the test substance. $¥$ Comparison of data from each experimental group was made with that from saline group using analysis of variance with repeated measures.

at 20 min postinjection. Values subsequently stabilized at this level as shown in Fig. 1 and remained relatively unchanged for an additional $30 \mathrm{~min}$ in the eight animals who were followed for a total of $60 \mathrm{~min}$ after the injection. Beginning $5 \mathrm{~min}$ postinjection, hematocrits were significantly higher in protamine sulfate-treated than in control animals as shown in Fig. 1.

Both hexadimethrine and poly-l-lysine induced changes in hematocrit similar to those observed with protamine sulfate and significantly different from those of the control group (Table I). The effect was observed more rapidly with poly-l-lysine than with the other polycations.

Plasma albumin concentration. Plasma albumin concentration showed no significant change during the entire period of study in the control rats who were given normal saline (Table II). In contrast, in each of the groups of animals injected with a polycation, plasma albumin concentration fell significantly $(P<0.0001)$. Most of this decline occurred within $8 \mathrm{~min}$ after injecting the polycation. For the 21 polycation-treated animals, plasma albumin concentration averaged $3.8 \mathrm{~g} / \mathrm{dl}$ before injection; $30 \mathrm{~min}$ after injection of the polycation, the value had fallen $>20 \%$ to $3.0 \mathrm{~g} / \mathrm{dl}$.

Albumin remaining in vascular space. The percentage of plasma albumin that remained in the vascular space after injection of the test substances was calculated from the preceding data. In the saline-injected control animals, intravascular albumin content remained constant throughout the $30 \mathrm{~min}$ of observation (Fig. 2). The injection of protamine sulfate was followed by a rapid and significant $(P<0.0001)$ decrease in albumin content of the vascular space. Values decreased to $56.0 \pm 11.7 \%$ within $5 \mathrm{~min}$ of injection and stabilized at $\sim 40 \%$ after $11 \mathrm{~min}$ after the injection (Fig. 2, Table III).

Statistically significant $(P<0.0001)$ losses of albumin from the vascular space also occurred after the injection of either hexadimethrine or poly-l-lysine (Table III).

${ }^{125}$ I-Albumin. Since each of the polycations had comparable effects, subsequent studies compared the effects of only protamine sulfate to those of saline. In the eight animals injected with normal saline, all the labeled albumin remained in the vascular space (Fig. 3). There was a dramatic loss of ${ }^{125} \mathrm{I}$-albumin from the vascular space after injection of protamine sulfate (Fig. 3 ). The magnitude and pattern of this loss was virtually identical to that described for endogenous albumin (Fig. 2). Experiments performed on six additional rats, three injected with saline and three with protamine sulfate, demonstrated that results $90 \mathrm{~min}$ after these injections were not different from those observed 30 min after the injections.

The use of labeled albumin allowed estimation of apparent volumes of distribution for albumin both before and after injections of either saline or protamine sulfate. This permitted the influence of protamine sulfate to be analyzed independently of change in hematocrit. Results are shown in Table IV. The volumes of distribution of albumin were not significantly dif-

Table II. Effect of an Intravenous Injection of either Saline or a Polycation on Plasma Albumin Concentration

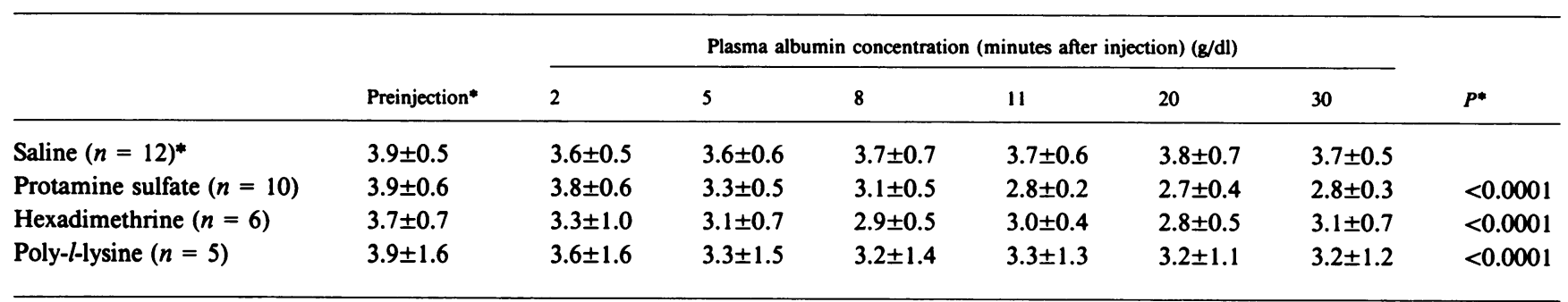

Values are given in mean \pm SD. * Details are as in Table $\mathbf{I}$. 


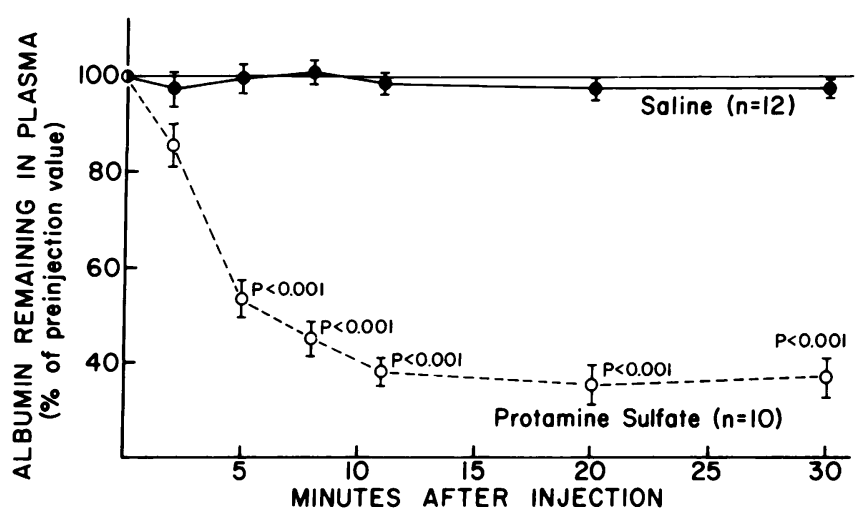

Figure 2. The effect of a protamine sulfate injection on the albumin content of the vascular space. Details as in Fig. 1.

ferent in the saline and protamine sulfate-injected groups of rats immediately before these injections $(5.96 \pm 0.67$ and $5.73 \pm 1.32 \mathrm{ml} / 100 \mathrm{~g}$ body $\mathrm{wt}$ respectively; $P>0.10, t$ test). Volume of distribution remained constant in the rats injected with saline. It increased significantly, by $\sim 35 \%$, after protamine sulfate. Most of this increase occurred within the first $8 \mathrm{~min}$ after injection.

${ }^{14} \mathrm{C}$-neutral dextran (Fig. 4). Even though $30 \mathrm{~min}$ was allowed for initial mixing of the dextran before any samples of blood were obtained, there was a progressive decline in the concentration of ${ }^{14} \mathrm{C}$-dextran in the plasma of the control rats injected with saline. Thus, only $56.3 \pm 15.3 \%$ of the amount of dextran present at the beginning of observations remained in the vascular space $60 \mathrm{~min}$ later. The relationship between the percentage of dextran remaining in the vascular space, plotted on a logarithmic scale against time, was linear $(r=0.997 ; P<0.001)$. The injection of protamine sulfate was followed by an increased rate of loss of dextran from the vascular space. The major effect was observed during the first $11 \mathrm{~min}$ after the injection and was statistically significant as shown in Fig. 4.

The loss of dextran from the vascular space attributable to the action of protamine sulfate was then calculated. Values for

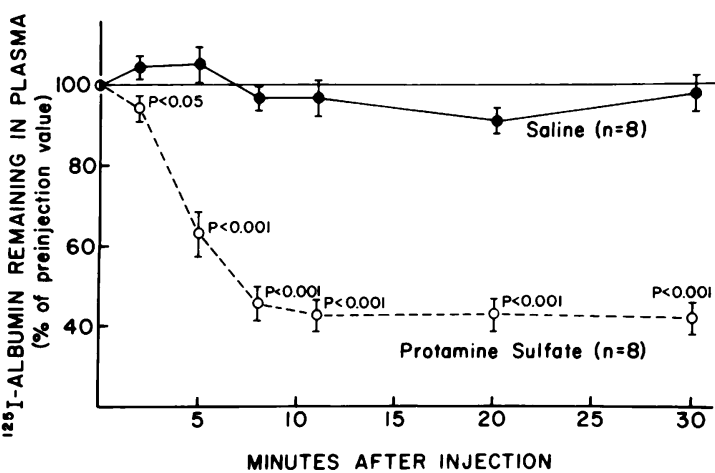

Figure 3. Changes in the ${ }^{125}$ I-albumin content of the vascular space after injecting either protamine sulfate or normal saline. Details as in Fig. 1.

the saline and protamine sulfate rats were first recalculated in a manner analogous to that for endogenous and ${ }^{125} \mathrm{I}$-albumin. Thus, postinjection observations were expressed as a percentage of the immediate preinjection value. The values from animals injected with protamine sulfate were then subtracted from those in animals injected with saline only. These differences are shown in Fig. 5, where the effects of protamine sulfate on vascular content of neutral dextran, molecular radius $36 \AA$, were compared with similar data for albumin. Since the values using endogenous and ${ }^{125} \mathrm{I}$-albumin were not different from one another, they were analyzed as a single group for these calculations.

At any time point the protamine sulfate-induced loss of albumin from the vascular space was significantly greater than the equivalent loss of dextran. The mean of values at 11,20 , and $30 \mathrm{~min}$ postinjection showed that the injection of protamine sulfate caused an average loss of $64.5 \%$ of albumin from the vascular space and that the equivalent loss of dextran was only $34.0 \%$.

Effects of Benadryl. Pretreatment of animals $(n=7)$ with Benadryl did not prevent an increase in hematocrit or a decrease in serum albumin concentration after the injection of protamine sulfate. During the first $8 \mathrm{~min}$ after protamine sulfate, the loss

Table III. Percentage of Intravascular Albumin Remaining in the Vascular Space at Varying Times after the Intravenous Injection of either Saline or a Polycation

\begin{tabular}{|c|c|c|c|c|c|c|c|}
\hline & \multicolumn{6}{|c|}{ Albumin content (minutes after injection) } & \multirow[b]{2}{*}{$P^{*}$} \\
\hline & 2 & 5 & 8 & 11 & 20 & 30 & \\
\hline & \multicolumn{6}{|c|}{ \% of preinjection value } & \\
\hline Saline $(n=12)^{*}$ & $97.4 \pm 12.4$ & $99.7 \pm 10.7$ & $100.9 \pm 8.6$ & $98.6 \pm 6.9$ & $97.5 \pm 8.2$ & $97.2 \pm 6.7$ & \\
\hline Protamine sulfate $(n=10)$ & $85.6 \pm 14.7$ & $53.2 \pm 11.4$ & $45.0 \pm 11.3$ & $38.0 \pm 9.1$ & $35.2 \pm 13.2$ & $36.8 \pm 13.1$ & $<0.0001$ \\
\hline Hexadimethrine $(n=6)$ & $97.7 \pm 11.9$ & $67.7 \pm 8.4$ & $54.5 \pm 9.6$ & $57.1 \pm 12.2$ & $47.9 \pm 5.0$ & $49.7 \pm 7.0$ & $<0.0001$ \\
\hline Poly-l-lysine $(n=5)$ & $78.3 \pm 13.4$ & $52.9 \pm 22.7$ & $51.7 \pm 17.8$ & $55.5 \pm 19.6$ & $53.9 \pm 18.5$ & $53.0 \pm 19.8$ & $<0.0001$ \\
\hline
\end{tabular}

Values are given in mean \pm SD. ${ }^{*}$ Details are as in Table $\mathrm{I}$. 
Table IV. Effect on the Apparent Volume of Distribution of ${ }^{125}$ I-Albumin of an Intravenous Injection of either Saline or Protamine Sulfate.

\begin{tabular}{|c|c|c|c|c|c|c|c|c|}
\hline & \multirow[b]{2}{*}{ Preinjection* } & \multicolumn{6}{|c|}{ Apparent volume of distribution (minutes after injection) } & \multirow[b]{2}{*}{$P^{*}$} \\
\hline & & 2 & 5 & 8 & 11 & 20 & 30 & \\
\hline & $\begin{array}{l}\mathrm{ml} / 100 \mathrm{~g} \\
\text { body wt }\end{array}$ & \multicolumn{6}{|c|}{$\%$ of control value } & \\
\hline Saline $(n=8)^{*}$ & $5.96 \pm 0.67$ & $102.7 \pm 5.6$ & $98.5 \pm 12.2$ & $106.1 \pm 12.0$ & $104.3 \pm 8.4$ & $102.6 \pm 5.4$ & $103.7 \pm 10.1$ & \\
\hline Protamine sulfate $(n=8)$ & $5.73 \pm 1.32$ & $99.8 \pm 7.9$ & $108.7 \pm 9.4$ & $125.2 \pm 10.8$ & $131.2 \pm 14.8$ & $135.6 \pm 15.0$ & $134.9 \pm 16.8$ & $<0.0001$ \\
\hline
\end{tabular}

Values are given in mean \pm SD. * Details as in Table I.

of albumin from the vascular space in these animals did not differ significantly from the loss in animals without pretreatment (Fig. 6). In both groups $\sim 50 \%$ of albumin was lost from the vascular space. No further loss, however, occurred in the animals pretreated with Benadryl; the loss of albumin continued in the animals not given the antihistamine. Thus, the loss of albumin at 11,20 , and $30 \mathrm{~min}$ was significantly less in the Benadrylpretreated animals than in those not given this drug. However, even in the Benadryl-pretreated animals, $43.2 \pm 13.5 \%$ of plasma albumin had been lost from the vascular space $30 \mathrm{~min}$ after

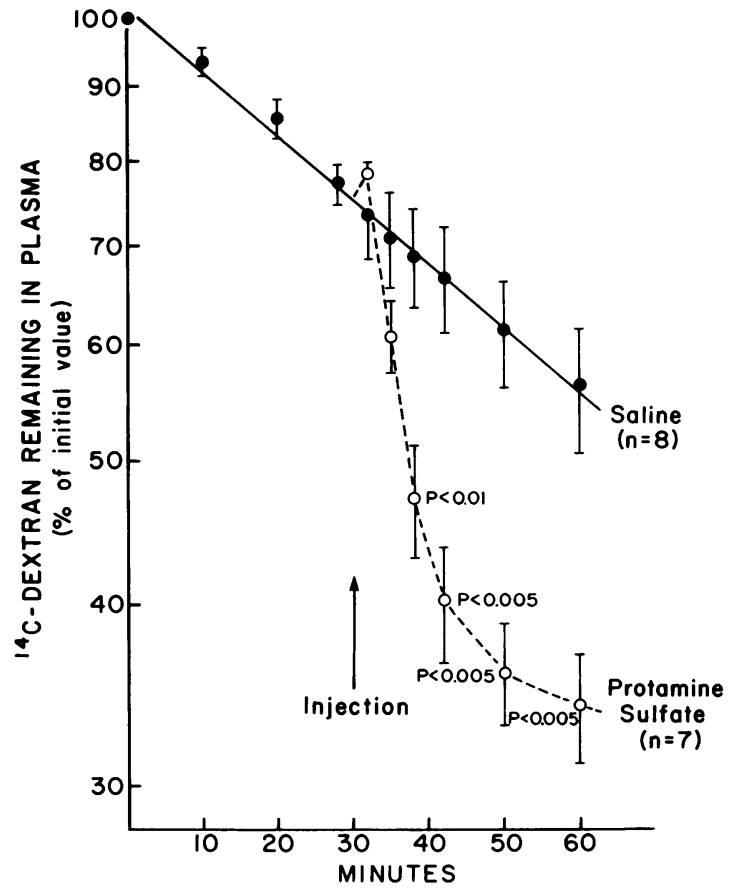

Figure 4. The percentages of ${ }^{14} \mathrm{C}$-labeled neutral dextran, comparable in size to albumin, that remained in the vascular space at varying time intervals during a control period of observation and following the intravenous injection of either saline or protamine sulfate. Details as in Fig. 1. protamine sulfate injection, compared with $67.3 \pm 13.9 \%$ in the animals not given this drug (Fig. 6).

Results from control animals $(n=5)$ pretreated with Benadryl and injected with normal saline were virtually identical to those, already presented, from control animals not pretreated with this drug. There were no statistically significant differences for values of hematocrit, plasma albumin concentration, or albumin remaining in the vascular space between these two groups of control animals.

Plasma protein characteristics before and after protamine sulfate. The administration of protamine sulfate did not modify the patterns of plasma electrophoresis on either polyacrylamide or SDS-polyacrylamide gel. Similarly the addition of protamine sulfate to rat serum albumin in vitro did not alter the migration of this protein in either polyacrylamide or SDS-polyacrylamide gel; nor did it alter the elution volume of albumin from Sephacryl S-200 columns.

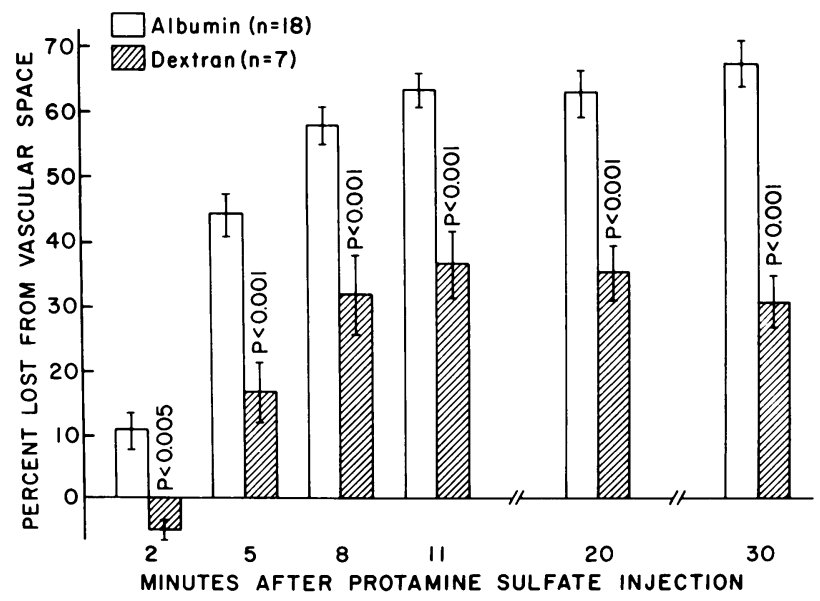

Figure 5. A comparison of the loss of albumin and neutral dextran of equal size, from the vascular space following injection of protamine sulfate. Values are shown as mean \pm SEM. The values for $P$ were calculated by the $t$ test. At any point in time the effect was greater on albumin than on dextran. 


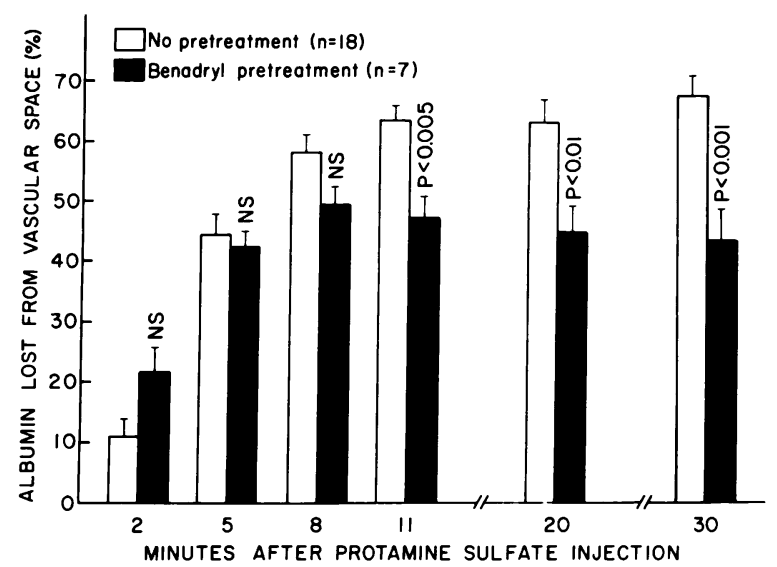

Figure 6. The effect of intravenous protamine sulfate on intravascular albumin content of untreated rats and those pretreated with Benadryl. Values are shown as mean \pm SEM. The values for $P$, calculated by the $t$ test, compare the two values at each time interval.

\section{Discussion}

The injection of each of the three polycations was followed within 10 min by a loss of $>50 \%$ of plasma albumin from the vascular space. Results using endogenous or ${ }^{125} \mathrm{I}$-labeled exogenous albumin were comparable. Simultaneously, hematocrit increased by $25 \%$, reflecting a decrease in plasma volume. If this shift of both fluid and albumin from the intravascular to the extravascular space was due to an increase in the transcapillary hydrostatic pressure gradient, the movement of water should have been proportionately greater than that of albumin. This was not the case, however. Rather than increasing, plasma albumin concentration decreased by $>20 \%$. Therefore, a decrease in the transcapillary colloid osmotic pressure gradient due to loss of protein from the vascular space appears to be the initiating event, followed by secondary movement of water to set a new balance between the hydrostatic and colloid osmotic pressures.

Our data do not permit a detailed analysis of whether the albumin loss was a generalized phenomenon or whether it occurred in only a limited number of capillary beds. The intravenous injection of one of the polycations we studied, poly-llysine, has been shown to induce pulmonary edema (25). It is unlikely, however, that the animals in our study could have survived if the losses of plasma fluid and albumin had occurred entirely into the lungs. The magnitude and the rapidity of the changes we observed suggest a generalized phenomenon. Indeed, polycations have been shown to increase the permeability of other capillary beds too, particularly in the skin and the mesentery (26).

There are several possible mechanisms to explain this increased escape of albumin across the walls of the microvasculature. One is that polycations might modify the physicochemical characteristics of albumin in a way that increases the ability of this protein to traverse the vascular walls. Our in vitro and in vivo studies of protein characteristics both before and after prot- amine sulfate administration did not provide any evidence to support such an argument.

A second explanation is that polycations stimulated the release of histamine, which in turn increased vascular permeability to albumin. As reviewed elsewhere (27) many agents including polylysine and protamine cause exocytic release of histamine, which increases vascular permeability and results in edema formation. The action of this autacoid is primarily on postcapillary venules where it causes contraction of endothelial cells. They separate at their boundaries and expose the basement membrane, which is permeable to albumin and water (27). Pretreating animals with Benadryl reduced the polycation-induced loss of albumin from the vascular space by less than one third. It failed to prevent most of the loss even though adequate doses of the drug were used (27). Higher doses of Benadryl did not provide any additional protection from the effects of protamine sulfate. Since it is the H1-blocking drugs that strongly antagonize the increased capillary permeability and edema formation due to histamine (27), histamine release can at most explain only a relatively small portion of the polycation-induced loss of protein from the vascular space.

It is now well accepted that in the renal glomerulus the capillary permeability to a given macromolecule depends, in addition to molecular size, on the charge of the molecule (12). We (15) and others (28) have demonstrated that in vivo administration of a polycation is followed by a decrease in negative charge sites in the glomerular capillaries and that this is accompanied by an increase in albuminuria. Enzymatic digestion of heparan sulfate, which is believed to be responsible for the fixed negative sites in the glomerular basement membrane, leads to similar changes (29). These effects could be a direct consequence of neutralization of the glomerular charge barrier, which would allow an increased filtration of albumin molecules that are negatively charged at $\mathrm{pH}$ 7.4. Such a mechanism would permit albuminuria to occur in the absence of a change in "pore size" of the glomerular basement membrane. An alternate explanation is that the negative charge sites are essential in maintaining the integrity of the basement membrane, and that neutralization leads to structural changes and to an increased pore size in the glomerular capillary filter. The two possibilities are not mutually exclusive; indeed some recent evidence suggests that there is an alteration in both size and charge selective barriers in the glomerulus after polycation treatment $(30,31)$.

The similarities of the present results to those obtained in the kidney are obvious. We propose that, as in the kidney, negative charge sites in the nonrenal capillary walls are important in maintaining the low permeability of these vessels to colloidal solutes. This is supported in the control animals by the observed difference in the distribution of charged albumin and that of neutral dextran of comparable size. While the labeled albumin was confined to the vascular space (Fig. 3, Table IV), there was a progressive loss from the vascular space of neutral dextran (Fig. 4). The choice of the dextran fraction that was used for these studies was based on its identical behavior to that of albumin on a Sephacryl column; this should eliminate any major 
differences in penetrance through vascular walls between the two species secondary to their differences in molecular shape and deformability. Thus, the difference can be best explained by discrimination based on molecular charge.

The changes induced by polycations further support the existence of a charge barrier in the nonrenal capillaries. We suggest that systemic injection of one of the polycations was followed by neutralization of the negative charge sites in capillary walls and that this permitted an increased movement of negatively charged albumin out of the vascular space, uncharged dextran being affected to a much lesser degree. The consequence of this loss of albumin and the hypoalbuminemia would be a decrease in plasma oncotic pressure, which in turn would result in a net loss of fluid from the vascular space and a marked decrease in plasma volume. This was reflected by hemoconcentration, as documented by an increase in hematocrit.

Whether the effects of polycations were due entirely to loss of charge without any change in the structure of the capillary walls, or whether an increase in pore size also played a role, is difficult to determine. Even if the primary event was an isolated increase in permeability to charged molecules, the transcapillary water flux secondary to decreased oncotic pressure would be associated with increased movement of neutral solutes by the well-documented phenomenon of solvent drag (5). On the other hand, loss of charge discrimination coupled with a small increase in pore size would result in similar changes, namely, the escape of albumin and water from the vascular space with a smaller increase in dextran movement.

The present study is not the first to identify an effect of polycations on vascular permeability $(25,26)$. However, the mechanisms responsible for these chanzes were not apparent at the time the earlier studies were published. Recent findings from other centers support our thesis that such changes could be mediated through alterations in charge sites in vessels and that charge may be important in maintaining the relative impermeability of the nonrenal microvasculature to albumin. In a perfusion study of the rabbit ear, dextran sulfate molecules were found to be less readily transported from the capillary lumen than were neutral dextrans (32). More recently, investigation of the rat hindquarter vascular bed has shown that the reflection coefficient for sulfated dextran is markedly higher than for neutral dextran (33).

Many tissues have been shown to contain polyanionic groups when stained appropriately. Indeed, the luminal front of blood vessel endothelial cells is negatively charged as are the subendothelial components that are exposed after removal of the endothelium (34). Furthermore, these anionic groups have the ability to redistribute by lateral migration under the influence of multivalent ligands. The addition of polycationic tracers results in rapid aggregation of most of these sites and leaves much of the luminal surface devoid of anionic sites (35). Furthermore, the charge of a molecule modifies its uptake and handling by the arterial endothelial cells (36). Permeability of artificial membranes may also be modified by alteration in charge, since the addition of protamine sulfate has been shown to increase the permeability of gelatin membranes to albumin (37).

In their study of solute exchange in the pulmonary circulation, Winn et al. (6) found that the observed data did not fit the typical mathematical models used to describe either a homoporous or a heteroporous membrane. One of their possible explanations for this discrepancy was that the electrical charge of a protein and of the microvascular wall may not be adequately described in the reflection and diffusion coefficients used in these mathematical analyses. Our findings suggest that this may well be the case. Thus, they add to the growing body of evidence that suggests that electrical charge may play a more prominent role in maintaining the integrity of all capillaries to albumin than has been appreciated previously.

Abnormalities of such a physiologic mechanism could represent an important, and previously unrecognized, cause for either generalized or localized edema. Recent observations have described endogenous substances that increase vascular permeability, but their mode of action is not understood $(38,39)$. It is possible that these agents result in cellular dysfunction, which causes loss of charge sites in capillary walls. Alternately, charge sites could be neutralized by these endogenous substances in a manner analogous to that proposed for polycations in the present studies. Thus, in addition to increased understanding of normal physiology, our findings may help to explain the pathophysiology of edema formation in a variety of disease processes.

\section{Acknowledgments}

We would like to express our appreciation to Michael Province, Division of Biostatistics, and to Dr. Marc Smith of the Health Administration Program, Washington University School of Medicine, for their assistance in the statistical analysis of the data. This project could not have been completed without the encouragement and support of Dr. Philip R. Dodge.

The study was supported by a grant from the Custom Music Corporation.

\section{References}

1. Landis, E. M., and J. R. Pappenheimer. 1963. Exchange of substances through the capillary walls. Handb. Physiol. 2:(Sect. 2). 9611034.

2. Starling, E. H. 1896. On the absorption of fluids from the connective tissue space. J. Physiol. (Lond.). 19:312-316.

3. Kedem, O., and A. Katchalsky. 1958. Thermodynamic analysis of the permeability of biological membranes to non-electrolytes. Biochim. Biophys. Acta. 27:229-246.

4. Patlack, C. S., D. A. Goldstein, and J. F. Hoffman. 1963. The flow of solute and solvent across a two membrane system. J. Theor. Biol. 5:426-442.

5. Katz, M. A. 1981. Changes in transcapillary protein flux by permeative and convective mechanisms as functions of increasing transcapillary water flux. Microvascular Res. 22:271-295.

6. Winn, R., B. Nadir, J. Gleisner, J. Stothert, and J. Hildebrandt. 
1981. Deficiencies in pore-membrane models of microvascular fluid and solute transudation. J. Appl. Physiol. 51:1574-1580.

7. Ichikawa, I., D. A. Maddox, M. G. Cogan, and B. M. Brenner. 1978. Dynamics of glomerular ultrafiltration in euvolemic Munich-Wistar rats. Renal Physiol. 1:121-131.

8. Brenner, B. M., W. M. Deen, and C. R. Robertson. 1976. Glomerular filtration. In The Kidney, B. M. Brenner and F. C. Rector, Jr., editors. W. B. Saunders Co., Philadelphia. 256.

9. Eisenbach, G. M., J. B. van Liew, and J. W. Boylan. 1975. Effect of angiotensin on the filtration of protein in the rat kidney: a micropuncture study. Kidney Int. 8:80-87.

10. Linker, A., P. Hovingh, Y. S. Kanwar, and M. G. Farquhar. 1981. Characterization of heparan sulfate isolated from dog glomerular basement membrane. Lab. Invest. 44:560-565.

11. Robson, A. M., J. Giangiacomo, R. A. Kienstra, S. T. Naqvi, and J. R. Ingelfinger. 1974. Normal glomerular permeability and its modification by minimal change nephrotic syndrome. J. Clin. Invest. 54:1190-1199.

12. Brenner, B. M., T. H. Hostetter, and H. D. Humes. 1978. Glomerular permselectivity: barrier function based on discrimination of molecular size and charge. Am. J. Physiol. 234:F455-F460.

13. Brenner, B. M., T. H. Hostetter, and H. D. Humes. 1978. Molecular basis of proteinuria of glomerular origin. N. Engl. J. Med. 298:826833.

14. Bridges, C. R., B. D. Myers, B. M. Brenner, and W. M. Deen. 1982. Glomerular charge alterations in human minimal change nephropathy. Kidney Int. 22:677-684.

15. Vehaskari, V. M., E. R. Root, F. G. Germuth, Jr., and A. M. Robson. 1982. Glomerular charge and urinary protein excretion: effects of systemic and intrarenal polycation infusion in the rat. Kidney Int. 22:127-135.

16. Shankel, S. W., A. M. Robson, and N. S. Bricker. 1967. On the mechanism of the splay in the glucose titration curve in advanced experimental renal disease in the rat. J. Clin. Invest. 46:164-172.

17. Mancini, G., A. O. Carbonara, and J. F. Heremans. 1965. Immunochemical quantitation of antigens by single radial immunodiffusion. Immunochemistry. 2:235-254.

18. Robson, A. M., J. Mor, E. R. Root, B. V. Jager, S. W. Shankel, J. R. Ingelfinger, R. A. Kienstra, and N. S. Bricker. 1979. Mechanisms of proteinuria in nonglomerular renal disease. Kidney Int. 16:416-429.

19. Fraker, P. J., and J. C. Speck, Jr. 1978. Protein and cell membrane iodinations with a sparingly soluble chloroamide, 1,3,4,6-tetrachloro$3 \alpha$,b $\alpha$-diphenyl-glycoluril. Biochem. Biophys. Res. Commun. 80:849857.

20. Mitruka, B. M., and H. M. Rawnsley. 1981. Clinical biochemical and hematological reference values in normal experimental animals and normal humans. Second ed. Masson Publishings USA, Inc., New York. 6.

21. Loening, V. E. 1967. The fractionation of high molecular weight ribonucleic acid by polyacrylamide gel electrophoresis. Biochem. J. 102:251-257.

22. Maizel, J. R., Jr. 1971. Polyacrylamide gel electrophoresis of viral proteins. Methods Virol. 5:179-246.
23. Statistical Analysis System (SAS) User's Guide. 1979. SAS Institute, Inc., Cary, NC. 237-263.

24. Armitage, P. 1973. Statistical Methods in Medical Research. John Wiley \& Sons, New York. 99-118.

25. deVries, A., J. O. Feldman, O. Stein, Y. Stein, and E. Katchalski. 1953. Effects of intravenously administered poly- $d$ l-lysine in rats. Proc. Soc. Exp. Biol. Med. 82:237-240.

26. Stein, O., A. deVries, and E. Katchalski. 1956. The effect of polyamino acids on the blood vessels of the rat. Arch. Int. Pharmacodyn. 17:243-253.

27. Douglas, W. W. 1980. Histamine and 5-hydroxytryptamine (serotonin) and their antagonists. In The Pharmacological Basis of Therapeutics. A. G. Gilman, L. S. Goodman, and A. Gilman, editors. Sixth ed. MacMillan Publishing Co., New York. 609-646.

28. Hunsicker, L. G., T. P. Shearer, and S. J. Shaffer. 1981. Acute reversible proteinuria induced by infusion of the polycation hexadimethrine. Kidney Int. 20:7-17.

29. Kanwar, Y. S., A. Linker, and M. G. Farquhar. 1980. Increased permeability of the glomerular basement membrane to ferritin after removal of glycosaminoglycans (heparan sulfate) by enzyme digestion. J. Cell Biol. 86:688-693.

30. Barnes, J. L., R. A. Radnik, E. P. Gilchrist, and M. A. Venkatachalam. 1982. Size and charge selective permeability defects induced in glomerular basement membrane by a polycation. Abstr. Amer. Soc Nephrol. 15:86A.

31. Bridges, C. R., H. G. Rennke, W. M. Deen, J. L. Troy, and B. M. Brenner. 1982. Reversible polycation-induced proteinuria. Abstr. Amer. Soc. Nephrol. 15:108A.

32. Areekul, S. 1969. Reflection coefficients of neutral and sulfatesubstituted dextran molecules in the isolated perfused rabbit ear. Acta Soc. Med. Upsal. 74:129-138.

33. Haraldsson, B., B. J. Moxham, and B. Rippe. 1982. Capillary permeability to sulfate-substituted and neutral dextran fractions in the rat hindquarter vascular bed. Acta Physiol. Scand. 115:397-404.

34. Skutelsky, E., Z. Rudich, and D. Danon. 1975. Surface charge properties of the luminal front of blood vessel walls: an electron microscopic analysis. Thromb. Res. 7:623-634.

35. Skutelsky, E., and D. Danon. 1976. Redistribution of surface anionic sites on the luminal front of blood vessel endothelium after interaction with polycation ligand. J. Cell Biol. 71:232-241.

36. Davies, P. F., H. G. Rennke, and R. S. Cotran. 1981. Influence of molecular charge upon the endocytosis and intracellular fate of peroxidase activity in cultured arterial endothelium. J. Cell Sci. 49:69-86.

37. Larsen, B. 1967. Increased permeability to albumin induced with protamine in modified gelatin membranes. Nature (Lond.). 215:641642.

38. Sobel, A., J. M. Heslan, A. Branellec, and G. Lagrue. 1981. Vascular permeability factor produced by lymphocytes of patients with nephrotic syndrome. Adv. Nephrol. 10:315-332.

39. Wedmore, C. V., and T. J. Williams. 1981. Control of vascular permeability by polymorphonuclear leukocytes in inflammation. Nature (Lond.). 289:646-650. 